\title{
microRNAs expression profiles in Schistosoma japonicum of different sex 14 and 28 days post-infection
}

\author{
Ai, L. ${ }^{1,2 \dagger}$, Hu, W. ${ }^{1,3 \#}$, Zhang, R.L. ${ }^{2}$, Huang, D.N. ${ }^{2}$, Chen, S.H. ${ }^{1}$, Xu, B. ${ }^{1}$, Li, H. ${ }^{1}$, Cai, Y.C. ${ }^{1}$, Lu, Y. ${ }^{1}$, \\ Zhou, X.N. ${ }^{1}$, Chen, M.X..$^{1,2,4^{*}}$ and Chen, J.X. ${ }^{1 *}$ \\ ${ }^{1}$ National Institute of Parasitic Diseases, Chinese Center for Disease Control and Prevention, \\ National Center for Tropical Diseases Research, WHO Collaborating Center for Tropical Diseases, \\ Key Laboratory of Parasite and Vector Biology, National Health and Family Planning Commission, \\ Shanghai 200025, PR China \\ ${ }^{2}$ Shenzhen Center for Disease Control and Prevention, Shenzhen 518055, PR China \\ ${ }^{3}$ Department of Microbiology and Microbial Engineering, School of Life Sciences, Fudan University, \\ Shanghai 200438, PR China \\ ${ }^{4}$ National Institute of Parasitic Diseases, Chinese Center for Disease Control and Prevention, \\ Shenzhen Center for Disease Control and Prevention Joint Laboratory for Imported Tropical Disease Control, \\ Shanghai, 200025, PR China \\ \#Ai, L. and $\mathrm{Hu}$, W. contributed equally to this work. \\ *Corresponding author e-mails: ipdchenmuxin@163.com (M.X. Chen); chenjiaxu1962@163.com (J.X. Chen) \\ Received 8 June 2020; received in revised form 14 September 2020; accepted 16 September 2020
}

\begin{abstract}
Different miRNAs are involved in the life cycles of Schistosoma japonicum. The aim of this study was to examine the expression profile of miRNAs in individual $S$. japonicum of different sex before and after pairing (18 and $24 \mathrm{dpi}$ ). The majority of differential expressed miRNAs were highly abundant at $14 \mathrm{dpi}$, except for sja-miR-125b and sja-miR-3505, in both male and female. Moreover, it was estimated that sja-miR-125b and sja-miR-3505 might be related to laying eggs. sja-miR-2a-5p and sja-miR-3484-5p were expressed at 14 dpi in males and were significantly clustered in DNA topoisomerase III, Rap guanine nucleotide exchange factor 1 and L-serine/L-threonine ammonia-lyase. Target genes of sja-miR-2d-5p, sja-miR-31$5 p$ and sja-miR-125a, which were expressed at 14 dpi in males but particularly females, were clustered in kelch-like protein 12, fructose-bisphosphate aldolase, class I, and heat shock protein $90 \mathrm{kDa}$ beta. Predicted target genes of sja-miR-3483-3p (expressed at 28 dpi in females but not in males) were clustered in $26 \mathrm{~S}$ proteasome regulatory subunit N1, ATPdependent RNA helicase DDX17. Predicted target genes of sja-miR-219-5p, which were differentially expressed at 28 dpi in females but particularly males, were clustered in DNA excision repair protein ERCC-6, protein phosphatase 1D, and ATPase family AAA domaincontaining protein $3 \mathrm{~A} / \mathrm{B}$. Moreover, at $28 \mathrm{dpi}$, eight miRNAs were significantly up-regulated in females compared to males. The predicted target genes of these miRNAs were significantly clustered in heat shock protein $90 \mathrm{kDa}$ beta, 26S proteasome regulatory subunit N1, and protein arginine N-methyltransferase 1 . To sum up, differentially expressed miRNAs may have an essential role and provide necessary information on clarifying this trematode's growth, development, maturation, and infection ability to mammalian hosts in its complex life cycle, and may be helpful for developing new drug targets and vaccine candidates for schistosomiasis.
\end{abstract}

\section{BACKGROUND}

Schistosomiasis, which is caused by the genus Schistosoma, is one of the most serious and debilitating parasitic diseases worldwide (Song et al., 2016). It has been reported that approximately 250 million people in 78 countries endemic with this disease, while nearly 800 million are at the risk of being infected (Gray et al., 2010; The Carter Center, 2014; WHO, 2016). In China, Schiatosomiasis japonica represents a serious public health problem (Li et al., 2014; Song et al., 2016; Sun et al., 2017). 
Currently, vaccines for schistosomiasis prevention are not available (Tebeje $e t$ al., 2016), and the only useful drug, i.e., praziquantel has an essential the role in treatment of schistosomiasis (Siqueira $e t$ al., 2017). However, the drug is ineffective against young worms (Wang et al., 2012). The life cycle of $S$. japonicum is complicated, with males and females pairing and eventually developing into adult worms in hosts. Given this condition, S. japonicum goes through remarkable morphological and molecular changes throughout its life cycle (Walker et al., 2011; Mourão et al., 2012; Sun et al., 2013). Previous studies have relied on genomic analysis technology to explain biology of $S$. japonicum and its interactions with hosts (S. japonicum Genome Sequencing and Functional Analysis Consortium, 2009), thus increasing our knowledge of microRNAs (miRNAs) and their evolutionary characteristics, developmental genetic switches, and novel biomarkers for S. japonicum. Consequently, a deeper understanding of the mechanisms of schistosome development and the pathogenesis of schistosomiasis may help developing novel strategies to control this disease.

Currently, different schistosome life cycle stages have been associated with different categories of miRNAs, suggesting that miRNAs may be involved in the regulation of development (Xue et al., 2008; Huang et al., 2009; Chen et al., 2010; Hao et al., 2010; Cai et al., 2011). Several lines of evidence have also indirectly implied that miRNAs may regulate the pathogenic schistosomiasis (Cheng et al., 2013). During the development of $S$. japonicum, males and females begin to pair approximately 14 days post-infection ( $14 \mathrm{dpi}$ ), and the female begins to lay eggs approximately 28 days post-infection (28 dpi) (He et al., 1980). Moreover, Sun et al. (2014) have reported a total of 2258 miRNAs identified in $S$. japonicum collected from single and doublesex infected mice; however, the authors did not provide information about the individual miRNAs before and after pairing (female at $14 \mathrm{dpi}$ and $28 \mathrm{dpi}$, male at $14 \mathrm{dpi}$ and $28 \mathrm{dpi}$ ).
In this study, the expression profiles of miRNAs in individual $S$. japonicum of different sex before and after pairing (18 dpi and $24 \mathrm{dpi}$ ) were investigated. Moreover, based on the analysis of the predicted targets, the different and specific functional requirements before and after pairing were determined based on the novel miRNA profiles.

\section{MATERIAL AND METHODS}

\section{Parasites}

Cercariae of $S$. japonicum were collected from the lab-infected snail $O$. hupensis hupensis, as well as hatched miracidia provided by the National Institute of Parasitic Disease, Chinese Center for Disease Control and Prevention (NIPD). Hepatic schistosomula were isolated from the portal system and from mesenteric veins of infected mice at $14 \mathrm{dpi}$. Adult worms of S. japonicum were isolated from the hepatic portal system and mesenteric veins of infected mice at $28 \mathrm{dpi}$. In addition, male and female adult worms were manually separated under a light microscope.

All animals were handled in strict accordance with the guidelines defined by the relevant national and/or local animal welfare bodies, and all animal experiments were approved by the ethics committee of NIPD (No. IPD-2018-16).

\section{RNA isolation}

According to the manufacturer's protocol, total RNAs from samples in female and male S. japonicum (schistosomulum and adult worm) at $14 \mathrm{dpi}$ and $28 \mathrm{dpi}$ were extracted using Trizol reagent (Invitrogen, Carlsbad, CA). RNA concentration and purity were photometrically measured at $260 \mathrm{~nm}$ and 280 nm using a NanoDrop ND-1000 spectrophotometer (Nanodrop Technologies, Wilmington, DE) and Agilent 2100 Bioanalyzer (Agilent Technologies, Palo Alto, $\mathrm{CA}$ ). The extracted RNA samples were then stored at $-80^{\circ} \mathrm{C}$. 
Small RNA isolation and library preparation

The cloning and analysis of small RNAs were performed according to the previous description (Ai et al., 2012; Xu et al., 2012). A $10 \mu \mathrm{g}$ total RNA was separated by using a Novex 15\% TBE-Urea gel (Invitrogen Co. Ltd). Then, 18-30 nt RNA was purified and the 5 ' and 3 ' adaptors (Illumina) were added to each end of the fragments. After each adaptor ligation, the small RNA was purified by using 6\% TBE PAGE gel. Complementary DNA (cDNA) was obtained by a reverse transcription PCR (RT-PCR) kit (Invitrogen) for reverse transcription. Finally, the RT-PCR product was purified by using a $6 \%$ TBE PAGE gel (Invitrogen) and sequenced with an Illumina-Solexa sequencer. Image and signal processing was completed using Solexa Genome Analysis System (Illumina) A file containing the trace, "base-calling" and quality score data were generated for subsequent bioinformatics analysis.

\section{Identification of non-coding RNA}

Clean reads were analyzed as previously described (Xu et al., 2010). Firstly, in order to obtain clean reads, adaptors and low-quality sequences were trimmed from the raw data file, as well as reads smaller than $18 \mathrm{nt}$ were removed. Then, the clean reads were searched against GenBank and Rfam database (version 10.1) to remove noncoding RNAs (rRNA, tRNA, snRNA, snoRNA) and other ncRNAs (Gardner et al., 2011). Repetitive sequences in the clean reads were eliminated by Repeat Masker (Bergman \& Quesneville, 2007) (http://www. repeatmasker.org). The Sanger miRBase (Kozomara A \& Griffiths-Jones, 2011) (version 16.0) was used to identify conserved miRNAs (Mount, 2007). The same miRNA family was judged according to reads showing high similarity to conserved miRNAs from other organisms with mismatches $\leq 2$ (Wei et al., 2009). The expression and coding characters of miRNAs were statistically analyzed by family distribution and nucleotide bias. "Unannotated" reads were those, which could not be matched using any of the databases. The genome of $S$. japonicum (http:// lifecenter.sgst.cn/schistosoma/cn) was used as the reference genome for short read alignment using the program Short Oligonucleotide Analysis Package (SOAP) (SOAP, http://soap.genomics.org.cn). The prediction of novel miRNAs was analyzed by Mfold (Zuker, 2008) and then evaluated with MirCheck (http://web.wi.mit.edu/bartel/pub/ software.html). The free energy hybridization threshold of a stem-loop hairpin was set lower than $-18 \mathrm{kcal} / \mathrm{mol}$.

\section{Target prediction and function analysis}

Algorithms Miranda was used to understand the molecular function of differentially expressed miRNAs. Moreover, TargetScan (Lewis et al., 2003) was used to get the putative target sites of miRNA candidates. The result of Miranda was filtered using the Energy of miRNA_target sites: -20, Score of miRNA_target sites: 150. Sequences predicted by both software were considered as miRNA targets.

To better understand miRNA target function and classification, all predicted target proteins with an $\mathrm{E}$ value of 1e-5 were identified by BLASTX searching against the Interpro and KEGG database (version 76). The best hits were used to validate the target gene function and metabolic pathway regulated by miRNAs. We also established the biological process, cellular component, and molecular function of target genes using the Interpro database the same way as we used the GO database.

\section{Analysis of miRNA expression}

In order to analyze novel miRNA expression at 14 dpi and 28 dpi of S. japonicum, RT-PCR with SYBR Green (TOYOBO) was used. RTPCR was performed using an ABI PRISM ${ }^{\circledR}$ 7300 Sequence Detection System and SYBR Green PCR Master Mix (TOYOBO) in a $20 \mu \mathrm{l}$ reaction. The PCR mix contained $5 \mu \mathrm{l}$ cDNA for each miRNA (in 1: 20 dilution), 5 $\mu \mathrm{M}$ forward and reverse primers, and $10 \mu \mathrm{l}$ $2 \times$ SYBR Green PCR Master Mix. The $S$. japonicum 18S rRNA gene (GenBank accession number L06668) was used as the endogenous control. The primer pairs for the RT-PCR are shown in Table 1. The PCR conditions were set at $95^{\circ} \mathrm{C} 10 \mathrm{~min}$, followed 
by 40 cycles of $95^{\circ} \mathrm{C}$ for $15 \mathrm{~s}, 65^{\circ} \mathrm{C}$ for $30 \mathrm{~s}$, and $72^{\circ} \mathrm{C}$ for $30 \mathrm{~s}$. The threshold cycle (Ct) was defined as the cycle number at which the fluorescence intensity passed a predetermined threshold value.

\section{Confirmation of miRNA expression by the reverse transcriptase-polymerase chain reaction}

To confirm the miRNA differential expressions at $14 \mathrm{dpi}$ and $28 \mathrm{dpi}$ of $S$. japonicum, stem-loop real-time reverse transcriptase-polymerase chain reaction (RT-PCR) with SYBR Green was applied. Real-time quantitative PCR was performed using an ABI PRISM ${ }^{\circledR} 7300$ Sequence Detection System and SYBR Green PCR Master Mix (TOYOBO) in a $20 \mu \mathrm{l}$ reaction. The PCR mix contained $5 \mu \mathrm{l}$ cDNA for each miRNA (in 1: 20 dilution), $5 \mu \mathrm{M}$ forward and reverse primers, $10 \mu \mathrm{l} 2 \times$ SYBR Green PCR Master Mix. The S. japonicum 18S rRNA gene (GenBank accession number L06668) was served as the endogenous control. The primers are shown in Table 1 . The PCR recycle conditions were set at $95^{\circ} \mathrm{C} 6 \mathrm{~min}$, followed by 50 cycles of $95^{\circ} \mathrm{C}$ for $10 \mathrm{~s}, 55^{\circ} \mathrm{C}$ for $10 \mathrm{~s}$, and $72^{\circ} \mathrm{C}$ for $30 \mathrm{~s}$. The threshold cycle (Ct) was defined as the cycle number at which the fluorescence intensity passed a predetermined threshold value.

\section{Statistical analysis}

At least three independent experiments were performed. Results were presented as mean \pm standard deviation. One-way ANOVA and Student's t-test were used to perform statistical analyses. A value of $\mathrm{P}<0.05$ was considered statistically significant.

\section{RESULTS}

Profile character of short RNAs from male and female Schistosomulum (14 dpi) Totally, 32,820,190 and 33,445,677 reads were obtained with Solexa deep sequencing from the female and the male samples of schistosomulum (14 dpi), respectively. After filtering low-quality tags, including 52 and 32 adaptors and contamination formed by adaptor-adaptor ligation, a total of 32,047,967 and 32,302,846 high-quality reads were obtained. Length distribution analysis showed that most reads were distributed among 20-23 nt in both genders. The highest percentage was $29.17 \%$ with reads that were $20 \mathrm{nt}$ long, which was followed by $23.08 \%$ of 23 nt reads in male (Fig. 1A), and $27.56 \%$ with 22 nt long reads, followed by $26.29 \%$ of $20 \mathrm{nt}$ reads in female (Fig.1B).

Among the clean reads, $2.59 \%$ and $2 \%$ were identified as noncoding sRNA, including tRNA, rRNA, siRNA, snRNA and snoRNA (Table 2) in male and female, respectively. Allowing the maximum mismatches of $2 \mathrm{nt}$, 4,155 (0.48\%) tRNAs, 15,905 (1.83\%) rRNAs, 241 (0.03\%) snoRNAs, and 2,156 (0.25\%) snRNA were recovered in Unique sRNAs of the male. In addition, 3,527 (0.42\%) tRNAs, 11,068 (1.33\%) rRNAs, 188 (0.02\%) snoRNAs, and $1,935(0.23 \%)$ snRNA were recovered in

Table 1. The primers used in identified novel miRNA expression at 14 dpi and 28 dpi of Schistosoma japonicum

\begin{tabular}{ll}
\hline Name of primers & Sequences (5' to $\left.3^{\prime}\right)$ \\
\hline 1.sja-miR-125b-RT & GTCGTATCCAGTGCGTGTCGTGGAGTCGGCAATTGCACTGGATACGACGCAATT \\
1.sja-miR-125b-F & TTCCCTGAGACTGATAATTG \\
2.sja-miR-10-5p-RT & GTCGTATCCAGTGCGTGTCGTGGAGTCGGCAATTGCACTGGATACGACAAACTC \\
2.sja-miR-10-5pF & AACCCTGTAGACCCGAGT \\
3.sja-miR-36-3p-RT & GTCGTATCCAGTGCGTGTCGTGGAGTCGGCAATTGCACTGGATACGACGCGAAT \\
3.sja-miR-36-3p-F & CACCGGGTAGACATTCATT \\
4.sja-miR-71a-RT & GTCGTATCCAGTGCGTGTCGTGGAGTCGGCAATTGCACTGGATACGACCATCTC \\
4.sja-miR-71a-F & GAAAGACGATGGTAGTGAG \\
5.sja-miR-3505-RT & GTCGTATCCAGTGCGTGTCGTGGAGTCGGCAATTGCACTGGATACGACAGCTAC \\
5.sja-miR-3505-F & TGACTGTCTGGACTCAGTA \\
6.sja-miR-let7-RT & GTCGTATCCAGTGCGTGTCGTGGAGTCGGCAATTGCACTGGATACGACACCACA \\
6.sja-miR-let7-F & GGAGGTAGTTCGTTGTGT \\
MiRNA primer-R & TGCGTGTCGTGGAGTCG \\
\hline
\end{tabular}


A

\section{Length Distribution}

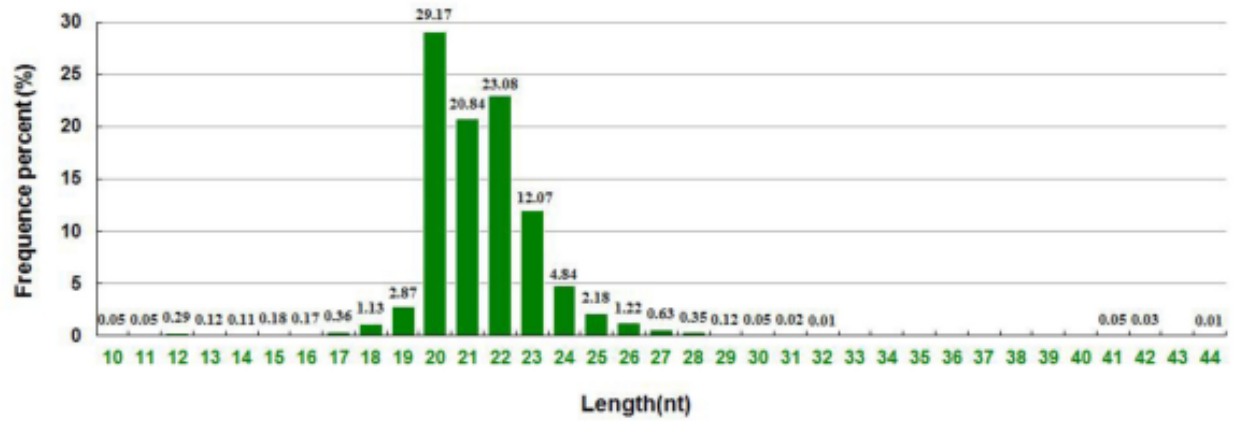

B

Length Distribution

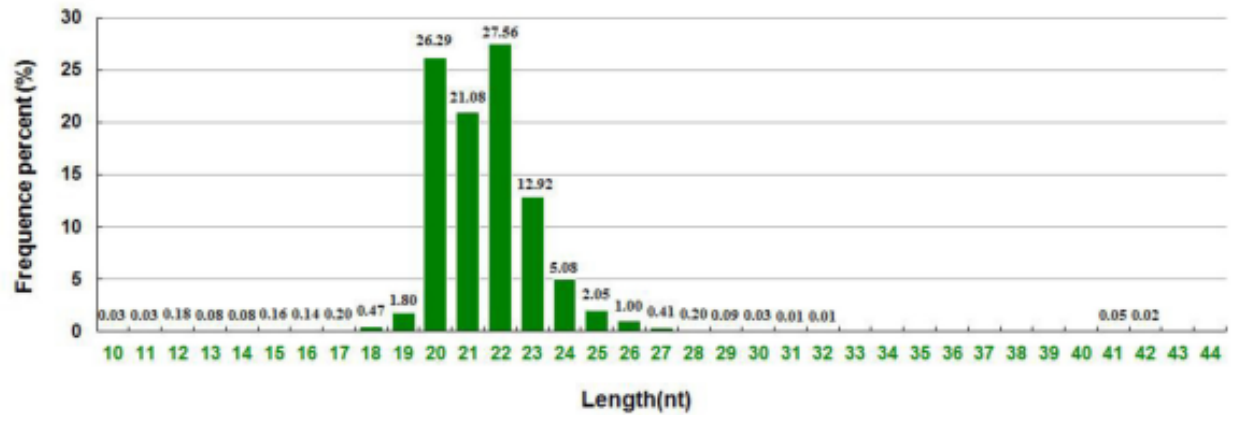

Figure 1. Length distribution analysis of small RNAs of male and female Schistosomulum (14 dpi). A resprents length distribution analysis of small RNAs of male Schistosomulum (14 dpi); B resprents length distribution analysis of small RNAs of female Schistosomulum (14 dpi).

Table 2. Small RNA annotation of 14 dpi male and female Schistosomulum

\begin{tabular}{|c|c|c|c|c|c|c|c|c|}
\hline \multirow{2}{*}{ Category } & \multicolumn{4}{|c|}{14 dpi Male } & \multicolumn{4}{|c|}{14 dpi Female } \\
\hline & $\begin{array}{l}\text { Unique } \\
\text { sRNAs }\end{array}$ & $\begin{array}{c}\text { Percent } \\
\text { (\%) }\end{array}$ & $\begin{array}{c}\text { Total } \\
\text { sRNAs }\end{array}$ & $\begin{array}{c}\text { Percent } \\
\text { (\%) }\end{array}$ & $\begin{array}{l}\text { Unique } \\
\text { sRNAs }\end{array}$ & $\begin{array}{l}\text { Percent } \\
\text { (\%) }\end{array}$ & $\begin{array}{c}\text { Total } \\
\text { sRNAs }\end{array}$ & $\begin{array}{c}\text { Percent } \\
(\%)\end{array}$ \\
\hline Total & 867552 & 100.00 & 29868057 & 100.00 & 831314 & 100.00 & 29711020 & 100.00 \\
\hline exon_antisense & 6067 & 0.70 & 384121 & 1.29 & 5515 & 0.66 & 357092 & 1.2 \\
\hline exon_sense & 10522 & 1.21 & 562133 & 1.88 & 8628 & 1.04 & 417787 & 1.41 \\
\hline intron_antisense & 8562 & 0.99 & 568474 & 1.9 & 8003 & 0.96 & 559974 & 1.88 \\
\hline intron_sense & 30872 & 3.56 & 1414260 & 4.74 & 26754 & 3.22 & 1059441 & 3.57 \\
\hline miRNA & 677 & 0.08 & 6173140 & 20.67 & 690 & 0.08 & 8024446 & 27.01 \\
\hline rRNA & 15905 & 1.83 & 1159093 & 3.88 & 11068 & 1.33 & 454410 & 1.53 \\
\hline repeat & 170547 & 19.66 & 6985139 & 23.39 & 154586 & 18.6 & 6350150 & 21.37 \\
\hline snRNA & 2156 & 0.25 & 40353 & 0.14 & 1935 & 0.23 & 28837 & 0.10 \\
\hline snoRNA & 241 & 0.03 & 2688 & 0.01 & 188 & 0.02 & 2111 & 0.01 \\
\hline tRNA & 4155 & 0.48 & 145993 & 0.49 & 3527 & 0.42 & 111156 & 0.37 \\
\hline unann & 617848 & 71.22 & 12432663 & 41.63 & 610420 & 73.43 & 12345616 & 41.55 \\
\hline
\end{tabular}


Unique sRNAs of the female. Except for the ncRNA mentioned above, 617,848 (71.22\%) and $610,420(73.43 \%)$ of total reads had no match to the public database and were marked as un-annotated reads (Table 2).

The level of the total percentage of ncRNA repressed in the schistosomulum of different sex (14 dpi) showed the following results: in males, 20,064,794 (67.18\%\%) sequences were perfectly mapped to the $S$. japonicum genome, which included 293,674 (33.85\%) unique sequences; in females, there were 19,615,952 (66.02\%) sRNAs mapping to the target genome, which contained 259,398 $(31.2 \% \%)$ unique sequences.

\section{Profile character of short RNAs from male and female adult worm of $S$. japonicum (28 dpi)}

Totally, 31,842,941 and 35,875,743 reads were obtained with solexa deep sequencing from the male and the female adult samples of S. japonicum (28 dpi), respectively. After filtering low-quality tags, including 52 and 32 adaptors and contamination formed by adaptor-adaptor ligation, a total of 31,636,846 and 35,680,733 high-quality reads were obtained. Length distribution analysis showed that most reads were distributed among 20-23 nt in both genders. The highest percentage was $42.69 \%$ with reads of $22 \mathrm{nt}$ long and followed by $18.12 \%$ of 21 nt reads in male (Fig. 2A), and it was $36.10 \%$ with reads of $22 \mathrm{nt}$ long and followed by $21.40 \%$ of $20 \mathrm{nt}$ reads in female (Fig. 2B).

Among the clean reads, $5.28 \%$ and $2.71 \%$ were identified as noncoding sRNA, including tRNA, rRNA, siRNA, snRNA and snoRNA (Table 3) in male and female, respectively. Allowing the maximum mismatches of $2 \mathrm{nt}$, 6,358 (1.21\%) tRNAs, 19,130 (3.65\%) rRNAs, $174(0.03 \%)$ snoRNAs, and 2,025 (0.39\%) snRNA were recovered in Unique sRNAs of the male. In addition, 5,403 (0.63\%) tRNAs, 15,744 (1.82\%) rRNAs, 168 (0.02\%) snoRNAs, and 2,072 (0.24\%) snRNA were recovered in

A

Length Distribution

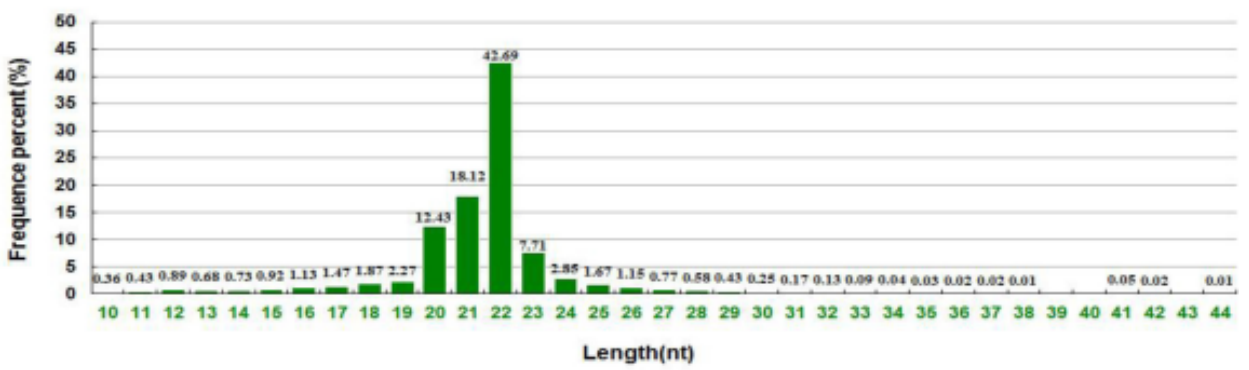

B

Length Distribution

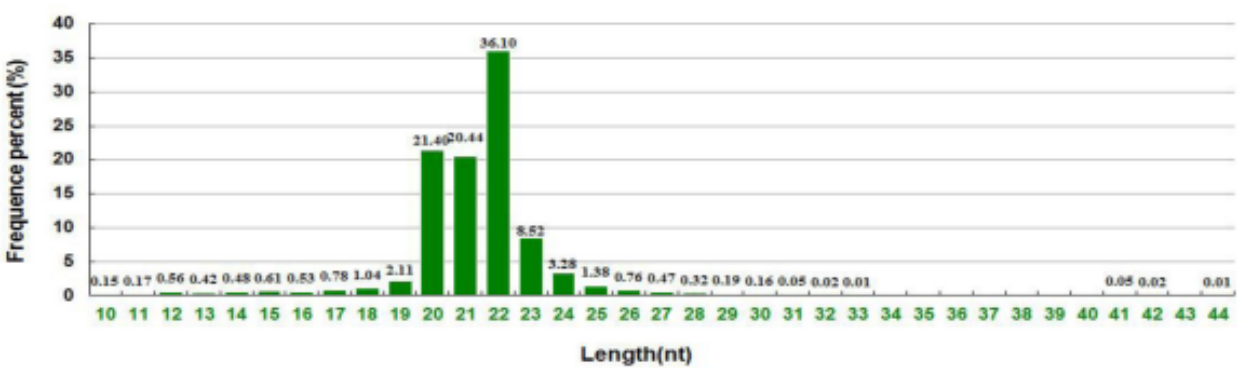

Figure 2. Length distribution analysis of small RNAs of male and female adult worm of Schistosoma japonicum (28 dpi). A resprents length distribution analysis of small RNAs of male adult worm of $S$. japonicum (28 dpi); B resprents length distribution analysis of small RNAs of female adult worm of $S$. japonicum (28 dpi). 
Table 3. Small RNA annotation of 28 dpi male and female adult worm of Schistosoma japonicum

\begin{tabular}{|c|c|c|c|c|c|c|c|c|}
\hline \multirow{2}{*}{ Category } & \multicolumn{4}{|c|}{28 dpi Male } & \multicolumn{4}{|c|}{28 dpi Female } \\
\hline & $\begin{array}{l}\text { Unique } \\
\text { sRNAs }\end{array}$ & $\begin{array}{c}\text { Percent } \\
(\%)\end{array}$ & $\begin{array}{c}\text { Total } \\
\text { sRNAs }\end{array}$ & $\begin{array}{l}\text { Percent } \\
\text { (\%) }\end{array}$ & $\begin{array}{l}\text { Unique } \\
\text { sRNAs }\end{array}$ & $\begin{array}{l}\text { Percent } \\
\text { (\%) }\end{array}$ & $\begin{array}{c}\text { Total } \\
\text { sRNAs }\end{array}$ & $\begin{array}{c}\text { Percent } \\
\text { (\%) }\end{array}$ \\
\hline Total & 524099 & 100.00 & 27150428 & 100.00 & 864237 & 100 & 32402321 & 100 \\
\hline exon_antisense & 2945 & 0.56 & 142862 & 1.29 & 5304 & 0.61 & 284633 & 0.88 \\
\hline exon_sense & 8560 & 1.63 & 336029 & 1.88 & 10156 & 1.18 & 478988 & 1.48 \\
\hline intron_antisense & 4285 & 0.82 & 260818 & 1.9 & 8195 & 0.95 & 575156 & 1.78 \\
\hline intron_sense & 16955 & 3.24 & 667169 & 4.74 & 27132 & 3.14 & 1003491 & 3.1 \\
\hline miRNA & 442 & 0.08 & 13782722 & 20.67 & 585 & 0.07 & 12197289 & 37.64 \\
\hline rRNA & 19130 & 3.65 & 1041006 & 3.88 & 15744 & 1.82 & 630786 & 1.95 \\
\hline repeat & 73833 & 14.09 & 2240278 & 23.39 & 146829 & 16.99 & 5535202 & 17.08 \\
\hline snRNA & 2025 & 0.39 & 36906 & 0.14 & 2072 & 0.24 & 27792 & 0.09 \\
\hline snoRNA & 174 & 0.03 & 2483 & 0.01 & 168 & 0.02 & 1608 & 0 \\
\hline tRNA & 6358 & 1.21 & 275372 & 0.49 & 5403 & 0.63 & 286893 & 0.89 \\
\hline unann & 389392 & 74.3 & 8364783 & 41.63 & 642649 & 74.36 & 11380483 & 35.12 \\
\hline
\end{tabular}

Unique sRNAs of the female. Except for the ncRNA mentioned above, 389,392 (74.3\%) and 836,4783 (41.63\%) of total reads had no match to the public database and were marked as un-annotated reads (Table 3).

The level of the total percentage of ncRNA repressed in different gender of adult $S$. japonicum (28 dpi) showed the following results: in males, 19,682,739 (72.5\%) sequences were perfectly mapped to the $S$. japonicum genome, which included 143,968 (27.47\%) unique sequences; in females, there were $22,860,844$ (70.55\%) sRNAs mapping to the target genome, which contained 254,308 (29.43\%) unique sequences.

Common and specific short RNAs sequences in schistosomulum (14 dpi) and adult worm of $S$. japonicum (28 dpi) Among the high-quality reads, 94.89\%, $90.62 \%, 96.90 \%$, and $91.56 \%$ of the total reads were shared by females at $14 \mathrm{dpi}$ and $28 \mathrm{dpi}$, males at $14 \mathrm{dpi}$ and $28 \mathrm{dpi}$, males at $14 \mathrm{dpi}$ and females at $14 \mathrm{dpi}$, and females and males at $28 \mathrm{dpi}$, respectively (Table 4 ). In the comparison between females at $14 \mathrm{dpi}$ and $28 \mathrm{dpi}$, there were 1.39 million (2.25\%) and 1.77 million (2.86\%) specific sRNAs expressed, respectively. Meanwhile, the comparison between males at $14 \mathrm{dpi}$ and 28 dpi, males and females at $14 \mathrm{dpi}$, as well as females and males at $28 \mathrm{dpi}$, the specific
sRNAs in each sample successively was 3.20 (5.61\%), 2.15 (3.77\%), 0.98 (1.65\%), 0.86 (1.45\%), 2.06 (4.98\%) and 2.97 (3.45\%) million.

\section{Known microRNA in schistosomulum (14 dpi) and adult worm of $S$. japonicum (28 dpi)}

The known miRNAs expressions in schistosomulum (14 dpi) and adult worm of $S$. japonicum (28 dpi) were shown in Table 5. There were $30,31,25$, and 27 known miRNAs in males and females at $14 \mathrm{dpi}$, and in males and females at $28 \mathrm{dpi}$, respectively. The number of miRNA precursors was 51, 51, 45 , and 49 in each sample, respectively. Moreover, there was no miRNA star (miRNA*) found in the above four specimens (Table 5).

Novel miRNA precursor candidates of schistosomulum (14 dpi) and adult worm of $S$. japonicum ( $28 \mathrm{dpi}$ )

The total number of novel miRNA precursor candidates of shistosomulum (14 dpi) and adult worm of $S$. japonicum (28 dpi) were 47477, 58541, 22617 and 33979 (in males at $14 \mathrm{dpi}$, females at $14 \mathrm{dpi}$, females and males at $28 \mathrm{dpi}$ ), respectively (Table 4). Moreover, the number of unique miRNA candidates were 464, 480, 454, and 336 in males at 14 dpi, females at $14 \mathrm{dpi}$, females and males at 28 dpi), respectively (Table 6). 


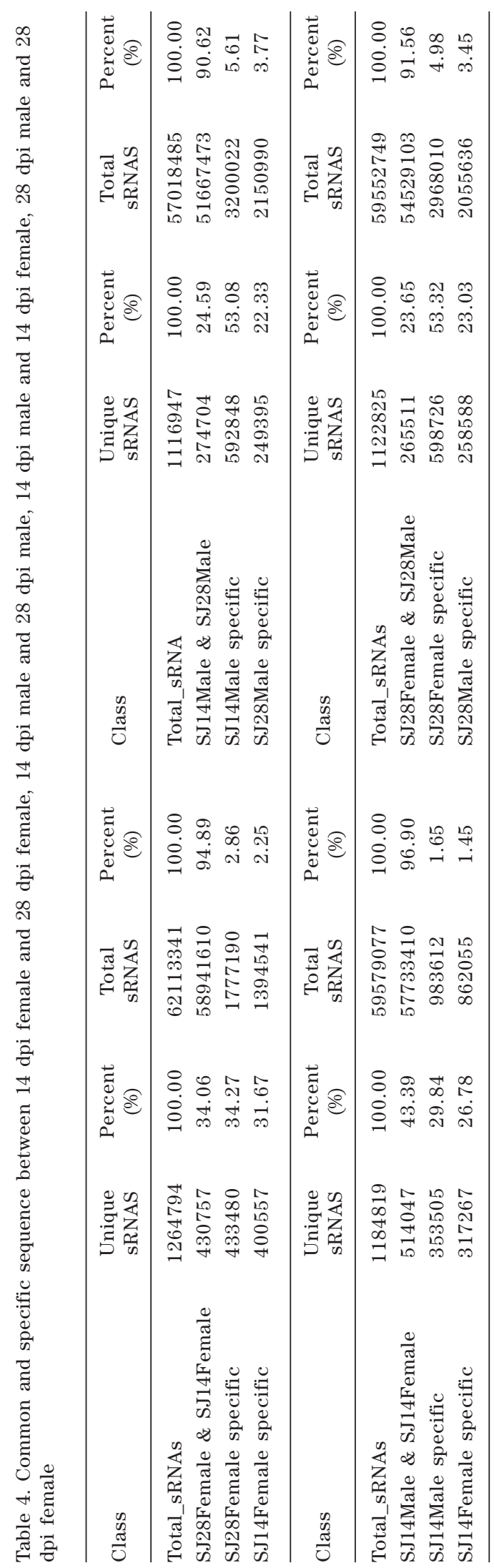

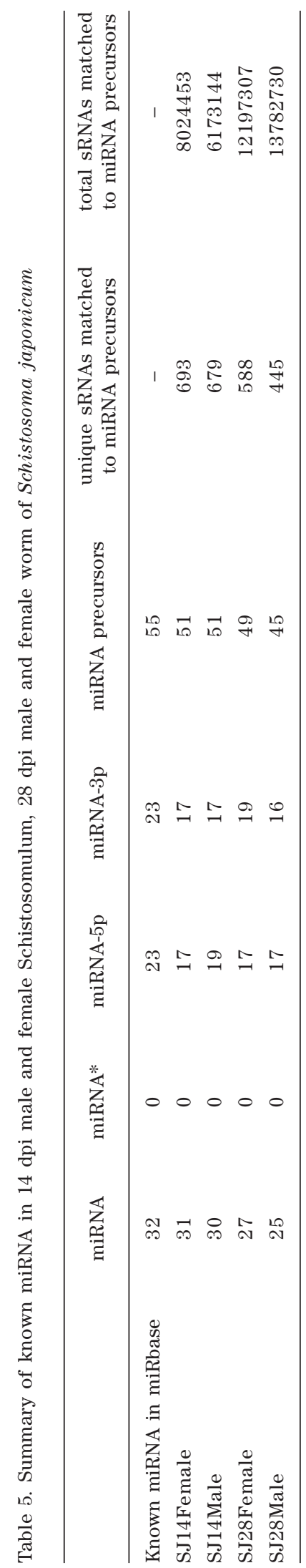


Table 6. Novel miRNA precursor candidates of each sample

\begin{tabular}{lcc}
\hline Sample & $\begin{array}{c}\text { Number of } \\
\text { unique miRNA } \\
\text { candidates }\end{array}$ & $\begin{array}{c}\text { Number of } \\
\text { total miRNA } \\
\text { candidates }\end{array}$ \\
\hline SJ14Female & 464 & 47477 \\
SJ14Male & 480 & 58541 \\
SJ28Female & 454 & 22617 \\
SJ28Male & 336 & 33979 \\
\hline
\end{tabular}

Differential expression analysis of schistosomulum (14 dpi) and adult worm of $S$. japonicum ( $28 \mathrm{dpi})$

There were 5 and 9 significantly differentially expressed miRNAs between mals and females at 14 dpi and 28 dpi, respectively. At 14 dpi, sja-miR-2a-5p and sja-miR-3484-5p were expressed only in male, while three miRNAs (sja-miR-2d-5p, sja-miR-31-5p and sja-miR-125a) showed high expression in females (Table 7). In females and males at 28 dpi male, except for sja-miR-219, other eight miRNAs (sja-miR-2c-5p, sja-miR-34833p, sja-miR-3489, sja-miR-3492, sja-miR3495, sja-miR-3499, sja-miR-3502, and sjamiR-3505) were highly expressed in females, and 9 miRNAs were differentially compared with those at $14 \mathrm{dpi}$.

Compared with both females and males at $28 \mathrm{dpi}$, the majority of differential expressed miRNAs were highly abundant at 14 dpi, except for sja-miR-125b and sja-miR3505 , which may be related to laying eggs process. These data suggested that more miRNAs have been actively expressed at 14 dpi in both male and female (Mark: begin to paired). In addition, there were 3 femalebiased expressions and 15 male-biased expressions in miRNAs at $14 \mathrm{dpi}$ and $28 \mathrm{dpi}$ (Table 7 and Table 8). We found that sja-let-7 was highly-expressed at 14 dpi in both males and females. Moreover, sja-bantam was expressed at different stages in males and females; its highest expression was observed at 28dpi in females.

Schistosomulum and adult worm of $S$. japonicum genes targeted by $S$. japonicum miRNAs

The application of KEGG pathway analysis was used to investigate the characteristics of the biochemical and metabolic processes involved in schistosomulum and adult worm of $S$. japonicum genes targeted by $S$. japonicum miRNAs. There was a significant difference in the expression of miRNAs, probably due to the function of sexual differentiation. We found the predicted target genes of sja-miR-2a-5p and sja-miR-3484-5p, which were expressed at 14 dpi in males and were clustered in DNA topoisomerase III, Rap guanine nucleotide exchange factor 1 and L-serine/L-threonine ammonia-lyase. Target genes of sja-miR-2d-5p, sja-miR-31-5p and sja-miR-125a, which were expressed at 14 dpi in male and female (mainly females) were clustered in kelch-like protein 12 , fructosebisphosphate aldolase, class I, and heat shock protein $90 \mathrm{kDa}$ beta. Predicted target genes of sja-miR-3483-3p (expressed at 28dpi in females without in the 28 dpi male) were clustered in $26 \mathrm{~S}$ proteasome regulatory subunit N1, ATP-dependent RNA helicase DDX17. Predicted target genes of sja-miR219-5p, which were differentially expressed at 28 dpi in male and female (mainly males) were clustered in DNA excision repair protein ERCC-6, protein phosphatase 1D, and ATPase family AAA domain-containing protein $3 \mathrm{~A} /$ B. At 28 dpi, eight miRNAs were significantly up-regulated in females compared to males, and the predicted target genes of these miRNAs were significantly clustered in heat shock protein $90 \mathrm{kDa}$ beta, $26 \mathrm{~S}$ proteasome regulatory subunit N1, and protein arginine N-methyltransferase 1.

Predicted target genes of these miRNAs, which revealed significantly high expression in females at both $14 \mathrm{dpi}$ and $28 \mathrm{dpi}$ were clustered in heat shock protein $90 \mathrm{kDa}$ beta (HSP90).

Based on the sequence similarity, clean reads but not the ncRNA or repeat sequences were blasted against known mature miRNAs dataset of $S$. japonicum in the public database of miRBase (Release 12.0) with the BLAST software (Altschul et al., 1990). Two co-expression and two differential expression miRNAs were identified. To verify the results of RNA-seq, the modified stem-loop RT-PCR was performed to examine the miRNA sjamiR-125b (the highest co-expression of female and male in 14 days), sja-miR-10-5p 


\begin{tabular}{|c|c|c|c|c|c|c|}
\hline pairwise & miR-name & SJ14Female-std & SJ14Male-std & $\begin{array}{c}\text { fold-change } \\
\text { (log2 SJ14Female/ } \\
\text { SJ14Male) }\end{array}$ & p-value & $\begin{array}{l}\text { sig- } \\
\text { lable }\end{array}$ \\
\hline SJ14Female.vs.SJ14Male & sja-bantam & 1332.25299 & 2070.62251 & -0.63619649 & 0.110738 & \\
\hline SJ14Female.vs.SJ14Male & sja-let-7 & 573.452912 & 515.836902 & 0.15276004 & 0.4158519 & \\
\hline SJ14Female.vs.SJ14Male & sja-miR-1 & 12.8869329 & 13.9616181 & -0.11555722 & 0.766251 & \\
\hline SJ14Female.vs.SJ14Male & sja-miR-10-3p & 93.8281046 & 71.2940278 & 0.39623889 & 0.00683229 & $* *$ \\
\hline SJ14Female.vs.SJ14Male & sja-miR-10-5p & 99531.0873 & 55267.7309 & 0.84870982 & 0.00585114 & $* *$ \\
\hline SJ14Female.vs.SJ14Male & sja-miR-124-3p & 99.2262629 & 89.8372855 & 0.14340768 & 0.2648972 & \\
\hline SJ14Female.vs.SJ14Male & sja-miR-124-5p & 13.8230297 & 8.38935368 & 0.72044228 & 0.04411403 & $*$ \\
\hline SJ14Female.vs.SJ14Male & sja-miR-125a & 252.309296 & 125.406907 & 1.00857656 & 0.00157489 & $* *$ \\
\hline SJ14Female.vs.SJ14Male & sja-miR-125b & 83842.6662 & 75990.3632 & 0.14186812 & 0.5467651 & \\
\hline SJ14Female.vs.SJ14Male & sja-miR-133 & 11.3267715 & 8.63700988 & 0.39113286 & 0.1073099 & \\
\hline SJ14Female.vs.SJ14Male & sja-miR-190-3p & 848.727784 & 605.302703 & 0.48764511 & 0.1136886 & \\
\hline SJ14Female.vs.SJ14Male & sja-miR-190-5p & 50.0187734 & 51.6053601 & -0.04505124 & 0.8458558 & \\
\hline SJ14Female.vs.SJ14Male & sja-miR-2162-3p & 1820.08425 & 2018.15035 & -0.14902843 & 0.2065674 & \\
\hline SJ14Female.vs.SJ14Male & sja-miR-2162-5p & 55.9473866 & 40.120304 & 0.47973821 & 0.00118765 & $* *$ \\
\hline SJ14Female.vs.SJ14Male & sja-miR-219-5p & 82.0020814 & 90.1158988 & -0.13612113 & 0.4504001 & \\
\hline SJ14Female.vs.SJ14Male & sja-miR-277 & 767.942628 & 920.414257 & -0.2612848 & 0.3615372 & \\
\hline SJ14Female.vs.SJ14Male & sja-miR-2a-3p & 4703.57449 & 3923.95766 & 0.26144808 & 0.00182198 & $* *$ \\
\hline SJ14Female.vs.SJ14Male & sja-miR-2a-5p & 0.01 & 0.3714843 & -5.21522932 & 0.01619183 & $*$ \\
\hline SJ14Female.vs.SJ14Male & sja-miR-2b-3p & 99.9751404 & 119.679857 & -0.25953905 & 0.2787144 & \\
\hline SJ14Female.vs.SJ14Male & sja-miR-2b-5p & 276.273375 & 212.612845 & 0.37786777 & 0.09236782 & \\
\hline SJ14Female.vs.SJ14Male & sja-miR-2c-3p & 847.074013 & 857.354798 & -0.01740433 & 0.862755 & \\
\hline SJ14Female.vs.SJ14Male & sja-miR-2c-5p & 6038.26133 & 4450.13421 & 0.44028435 & 0.1365844 & \\
\hline SJ14Female.vs.SJ14Male & sja-miR-2d-3p & 2136.57859 & 1731.9217 & 0.30292867 & 0.00016955 & $* *$ \\
\hline SJ14Female.vs.SJ14Male & sja-miR-2d-5p & 1.31053555 & 0.18574215 & 2.81878336 & 0.00781897 & $* *$ \\
\hline SJ14Female.vs.SJ14Male & sja-miR-2e-3p & 55.3857285 & 50.9862196 & 0.11940691 & 0.213092 & \\
\hline SJ14Female.vs.SJ14Male & sja-miR-2e-5p & 2.30903882 & 1.98124958 & 0.2208818 & 0.6192086 & \\
\hline SJ14Female.vs.SJ14Male & sja-miR-307 & 374.313915 & 376.74699 & -0.0093473 & 0.9683188 & \\
\hline SJ14Female.vs.SJ14Male & sja-miR-310 & 0.49925164 & 0.74296859 & -0.57353406 & 0.3013142 & \\
\hline SJ14Female.vs.SJ14Male & sja-miR-31-5p & 1.80978719 & 0.27861322 & 2.69948443 & 0.01016709 & $*$ \\
\hline SJ14Female.vs.SJ14Male & sja-miR-3479-3p & 236.052415 & 254.219086 & -0.10696511 & 0.4600628 & \\
\hline SJ14Female.vs.SJ14Male & sja-miR-3479-5p & 0.3432355 & 0.2476562 & 0.47086006 & 0.7114215 & \\
\hline SJ14Female.vs.SJ14Male & sja-miR-3481-3p & 1.06090973 & 1.05253884 & 0.01142844 & 0.9493682 & \\
\hline SJ14Female.vs.SJ14Male & sja-miR-3481-5p & 1.34173878 & 2.22890578 & -0.73223181 & 0.07201406 & \\
\hline SJ14Female.vs.SJ14Male & sja-miR-3482-3p & 1.87219364 & 2.97187437 & -0.66664347 & 0.1958045 & \\
\hline SJ14Female.vs.SJ14Male & sja-miR-3482-5p & 3.40115178 & 3.83867106 & -0.17458355 & 0.5745236 & \\
\hline SJ14Female.vs.SJ14Male & sja-miR-3483-3p & 0.3432355 & 0.2476562 & 0.47086006 & 0.7832769 & \\
\hline SJ14Female.vs.SJ14Male & sja-miR-3484-5p & 0.01 & 0.34052727 & -5.08969844 & 0.02426888 & $*$ \\
\hline SJ14Female.vs.SJ14Male & sja-miR-3486-5p & 0.43684518 & 0.71201157 & -0.7047786 & 0.1490595 & \\
\hline SJ14Female.vs.SJ14Male & sja-miR-3487 & 6.0534261 & 5.13886609 & 0.23630185 & 0.1615179 & \\
\hline SJ14Female.vs.SJ14Male & sja-miR-3488 & 0.21842259 & 0.1238281 & 0.81878336 & 0.7242039 & \\
\hline SJ14Female.vs.SJ14Male & sja-miR-3489 & 46.4928087 & 70.5820162 & -0.60229306 & 0.09538728 & \\
\hline SJ14Female.vs.SJ14Male & sja-miR-3490 & 0.12481291 & 0.2476562 & -0.98857156 & 0.49663 & \\
\hline SJ14Female.vs.SJ14Male & sja-miR-3491 & 0.21842259 & 0.40244132 & -0.88165636 & 0.3103331 & \\
\hline SJ14Female.vs.SJ14Male & sja-miR-3492 & 224.257595 & 229.855908 & -0.0355729 & 0.8688266 & \\
\hline SJ14Female.vs.SJ14Male & sja-miR-3493 & 0.01 & 0.06191405 & -2.63026682 & 0.373901 & \\
\hline SJ14Female.vs.SJ14Male & sja-miR-3494 & 0.40564196 & 0.65009752 & -0.68044927 & 0.5681987 & \\
\hline SJ14Female.vs.SJ14Male & sja-miR-3495 & 31.7336822 & 31.8547784 & -0.00549487 & 0.8822446 & \\
\hline SJ14Female.vs.SJ14Male & sja-miR-3496 & 0.56165809 & 0.89775371 & -0.67662756 & 0.484287 & \\
\hline SJ14Female.vs.SJ14Male & sja-miR-3497 & 0.06240645 & 0.01 & 2.64169525 & 0.373901 & \\
\hline SJ14Female.vs.SJ14Male & sja-miR-3498 & 0.62406455 & 0.61914049 & 0.01142844 & 0.9240003 & \\
\hline SJ14Female.vs.SJ14Male & sja-miR-3499 & 18.6283267 & 21.0507768 & -0.17637538 & 0.3421255 & \\
\hline SJ14Female.vs.SJ14Male & sja-miR-3500 & 0.56165809 & 0.52626942 & 0.0938906 & 0.9445722 & \\
\hline SJ14Female.vs.SJ14Male & sja-miR-3501 & 0.06240645 & 0.18574215 & -1.57353406 & 0.09287917 & \\
\hline SJ14Female.vs.SJ14Male & sja-miR-3502 & 2.43385173 & 2.69326114 & -0.14611284 & 0.741526 & \\
\hline SJ14Female.vs.SJ14Male & sja-miR-3503 & 0.06240645 & 0.01 & 2.64169525 & 0.373901 & \\
\hline SJ14Female.vs.SJ14Male & sja-miR-3504 & 1.15451941 & 1.85742148 & -0.68600879 & 0.1269994 & \\
\hline SJ14Female.vs.SJ14Male & sja-miR-3505 & 1.77858396 & 1.42402313 & 0.32075649 & 0.3260326 & \\
\hline SJ14Female.vs.SJ14Male & sja-miR-3506 & 2.09061623 & 1.70263636 & 0.29615791 & 0.3108077 & \\
\hline SJ14Female.vs.SJ14Male & sja-miR-3507 & 4.58687442 & 4.58163965 & 0.00164742 & 0.9711712 & \\
\hline SJ14Female.vs.SJ14Male & sja-miR-36-3p & 26208.9948 & 23184.6135 & 0.17689434 & 0.1669596 & \\
\hline SJ14Female.vs.SJ14Male & sja-miR-36-5p & 31.4216499 & 22.3819288 & 0.48942457 & 0.0569673 & \\
\hline SJ14Female.vs.SJ14Male & sja-miR-61 & 651.273761 & 617.71647 & 0.07631931 & 0.212293 & \\
\hline SJ14Female.vs.SJ14Male & sja-miR-71a & 13751.1687 & 12708.9421 & 0.11371029 & 0.2048831 & \\
\hline SJ14Female.vs.SJ14Male & sja-miR-71b-3p & 13.8854362 & 12.3828099 & 0.16523377 & 0.6965346 & \\
\hline SJ14Female.vs.SJ14Male & sja-miR-71b-5p & 3515.76123 & 3120.00373 & 0.17228934 & 0.182484 & \\
\hline SJ14Female.vs.SJ14Male & sja-miR-7-3p & 4.11882601 & 6.16044791 & -0.58080206 & 0.1706581 & \\
\hline SJ14Female.vs.SJ14Male & sja-miR-7-5p & 0.3432355 & 0.80488264 & -1.22957966 & 0.2568139 & \\
\hline SJ14Female.vs.SJ14Male & sja-miR-8-3p & 134.860349 & 139.058955 & -0.04423042 & 0.864476 & \\
\hline
\end{tabular}


Table 8. Differential expression analysis of adult male and female worm of Schistosoma japonicum (28 dpi)

\begin{tabular}{|c|c|c|c|c|c|c|}
\hline pairwise & miR-name & SJ28Female-std & SJ28Male-std & $\begin{array}{c}\text { fold-change } \\
\text { (log2 SJ28Female/ } \\
\text { SJ28Male) }\end{array}$ & p-value & $\begin{array}{l}\text { sig- } \\
\text { lable }\end{array}$ \\
\hline SJ28Female.vs.SJ28Male & sja-bantam & 7975.124764 & 691.571486 & 3.527556797 & 0.06601843 & \\
\hline SJ28Female.vs.SJ28Male & sja-let-7 & 155.5085 & 158.3551713 & -0.02617054 & 0.96442 & \\
\hline SJ28Female.vs.SJ28Male & sja-miR-1 & 2.826356683 & 3.262467871 & -0.20702015 & 0.9203389 & \\
\hline SJ28Female.vs.SJ28Male & sja-miR-10-3p & 34.65231058 & 25.20346058 & 0.459329715 & 0.5284895 & \\
\hline SJ28Female.vs.SJ28Male & sja-miR-10-5p & 87257.78602 & 128402.7405 & -0.55732022 & 0.1662015 & \\
\hline SJ28Female.vs.SJ28Male & sja-miR-124-3p & 23.22911899 & 27.13943053 & -0.22445601 & 0.8436504 & \\
\hline SJ28Female.vs.SJ28Male & sja-miR-124-5p & 0.765471602 & 1.649159583 & -1.10731025 & 0.3728084 & \\
\hline SJ28Female.vs.SJ28Male & sja-miR-125a & 435.6711062 & 637.7228404 & -0.54969012 & 0.1893544 & \\
\hline SJ28Female.vs.SJ28Male & sja-miR-125b & 245686.9135 & 349279.5234 & -0.50756105 & 0.3214888 & \\
\hline SJ28Female.vs.SJ28Male & sja-miR-133 & 2.855797898 & 2.473739374 & 0.207200375 & 0.7575477 & \\
\hline SJ28Female.vs.SJ28Male & sja-miR-190-3p & 90.35509021 & 153.586949 & -0.76537785 & 0.06505089 & \\
\hline SJ28Female.vs.SJ28Male & sja-miR-190-5p & 44.27958803 & 27.85645643 & 0.668630053 & 0.254794 & \\
\hline SJ28Female.vs.SJ28Male & sja-miR-2162-3p & 489.8723838 & 450.1130122 & 0.122118691 & 0.6795604 & \\
\hline SJ28Female.vs.SJ28Male & sja-miR-2162-5p & 15.83937391 & 6.919299989 & 1.194817315 & 0.1198851 & \\
\hline SJ28Female.vs.SJ28Male & sja-miR-219-5p & 44.19126439 & 99.95341124 & -1.17749459 & 0.01181171 & $*$ \\
\hline SJ28Female.vs.SJ28Male & sja-miR-277 & 568.7454 & 446.4203288 & 0.349380246 & 0.5862748 & \\
\hline SJ28Female.vs.SJ28Male & sja-miR-2a-3p & 928.5170527 & 443.8748869 & 1.064775319 & 0.1705301 & \\
\hline SJ28Female.vs.SJ28Male & sja-miR-2a-5p & 0.01 & 0.071702591 & -2.84202524 & 0.373901 & \\
\hline SJ28Female.vs.SJ28Male & sja-miR-2b-3p & 48.46024062 & 24.3071782 & 0.995419147 & 0.3208904 & \\
\hline SJ28Female.vs.SJ28Male & sja-miR-2b-5p & 232.7033669 & 113.4334983 & 1.036645336 & 0.1362916 & \\
\hline SJ28Female.vs.SJ28Male & sja-miR-2c-3p & 104.8401682 & 15.88212381 & 2.722715821 & 0.07153119 & \\
\hline SJ28Female.vs.SJ28Male & sja-miR-2c-5p & 517.6943324 & 128.9929604 & 2.004808189 & 0.03397996 & $*$ \\
\hline SJ28Female.vs.SJ28Male & sja-miR-2d-3p & 266.6196471 & 102.2478941 & 1.382711966 & 0.0820081 & \\
\hline SJ28Female.vs.SJ28Male & sja-miR-2d-5p & 0.058882431 & 0.01 & 2.557837233 & 0.373901 & \\
\hline SJ28Female.vs.SJ28Male & sja-miR-2e-3p & 19.60784949 & 13.26497925 & 0.563808896 & 0.489697 & \\
\hline SJ28Female.vs.SJ28Male & sja-miR-2e-5p & 0.471059447 & 0.286810362 & 0.715811989 & 0.3491035 & \\
\hline SJ28Female.vs.SJ28Male & sja-miR-307 & 107.3426715 & 93.75113716 & 0.195315604 & 0.7681738 & \\
\hline SJ28Female.vs.SJ28Male & sja-miR-310 & 0.147206077 & 0.107553886 & 0.452777583 & 0.6618122 & \\
\hline SJ28Female.vs.SJ28Male & sja-miR-31-5p & 0.588824309 & 0.107553886 & 2.452777583 & 0.2401411 & \\
\hline SJ28Female.vs.SJ28Male & sja-miR-3479-3p & 239.4748464 & 161.4742339 & 0.568570153 & 0.2895729 & \\
\hline SJ28Female.vs.SJ28Male & sja-miR-3479-5p & 0.117764862 & 0.01 & 3.557837233 & 0.1169925 & \\
\hline SJ28Female.vs.SJ28Male & sja-miR-3480-3p & 0.088323646 & 0.01 & 3.142799733 & 0.373901 & \\
\hline SJ28Female.vs.SJ28Male & sja-miR-3481-3p & 1.177648618 & 0.932133677 & 0.337300366 & 0.6149557 & \\
\hline SJ28Female.vs.SJ28Male & sja-miR-3481-5p & 0.883236463 & 0.143405181 & 2.622702585 & 0.0791651 & \\
\hline SJ28Female.vs.SJ28Male & sja-miR-3482-3p & 0.97156011 & 1.541605697 & -0.66605865 & 0.5668704 & \\
\hline SJ28Female.vs.SJ28Male & sja-miR-3482-5p & 0.294412154 & 2.652995851 & -3.17171328 & 0.1948922 & \\
\hline SJ28Female.vs.SJ28Male & sja-miR-3483-3p & 0.32385337 & 0.01 & 5.017268851 & 0.000232496 & $* *$ \\
\hline SJ28Female.vs.SJ28Male & sja-miR-3484-5p & 0.01 & 0.143405181 & -3.84202524 & 0.373901 & \\
\hline SJ28Female.vs.SJ28Male & sja-miR-3485-3p & 0.058882431 & 0.01 & 2.557837233 & 0.373901 & \\
\hline SJ28Female.vs.SJ28Male & sja-miR-3486-5p & 0.382735801 & 0.071702591 & 2.416251707 & 0.369566 & \\
\hline SJ28Female.vs.SJ28Male & sja-miR-3487 & 7.772480878 & 5.879612426 & 0.402654104 & 0.8020005 & \\
\hline SJ28Female.vs.SJ28Male & sja-miR-3488 & 0.529941878 & 0.143405181 & 1.885736991 & 0.06665169 & \\
\hline SJ28Female.vs.SJ28Male & sja-miR-3489 & 43.21970428 & 3.692683414 & 3.548947715 & 0.005475989 & $* *$ \\
\hline SJ28Female.vs.SJ28Male & sja-miR-3490 & 0.117764862 & 0.01 & 3.557837233 & 0.1169925 & \\
\hline SJ28Female.vs.SJ28Male & sja-miR-3491 & 0.294412154 & 0.01 & 4.879765328 & 0.1325751 & \\
\hline SJ28Female.vs.SJ28Male & sja-miR-3492 & 120.6206597 & 24.55813727 & 2.296203989 & 0.001458799 & $* *$ \\
\hline SJ28Female.vs.SJ28Male & sja-miR-3494 & 0.206088508 & 0.179256476 & 0.201238816 & 0.8846557 & \\
\hline SJ28Female.vs.SJ28Male & sja-miR-3495 & 37.39034362 & 8.138244029 & 2.199876284 & 0.02123222 & $*$ \\
\hline SJ28Female.vs.SJ28Male & sja-miR-3496 & 0.588824309 & 0.143405181 & 2.037740084 & 0.1606805 & \\
\hline SJ28Female.vs.SJ28Male & sja-miR-3499 & 27.58641887 & 1.147241449 & 4.587717227 & 0.002872435 & $* *$ \\
\hline SJ28Female.vs.SJ28Male & sja-miR-3500 & 0.294412154 & 0.143405181 & 1.037740084 & 0.4626249 & \\
\hline SJ28Female.vs.SJ28Male & sja-miR-3502 & 2.060885081 & 0.430215543 & 2.260132505 & 0.0262687 & $*$ \\
\hline SJ28Female.vs.SJ28Male & sja-miR-3503 & 0.01 & 0.179256476 & -4.16395334 & 0.1210549 & \\
\hline SJ28Female.vs.SJ28Male & sja-miR-3504 & 0.824354032 & 0.358512953 & 1.201238816 & 0.1076292 & \\
\hline SJ28Female.vs.SJ28Male & sja-miR-3505 & 10.83436728 & 0.322661658 & 5.069448944 & 0.02599001 & $*$ \\
\hline SJ28Female.vs.SJ28Male & sja-miR-3506 & 1.001001325 & 0.01 & 6.645300074 & 0.1337789 & \\
\hline SJ28Female.vs.SJ28Male & sja-miR-3507 & 2.355297236 & 2.294482898 & 0.037740084 & 0.9490775 & \\
\hline SJ28Female.vs.SJ28Male & sja-miR-36-3p & 9682.509171 & 9620.551683 & 0.009261336 & 0.986818 & \\
\hline SJ28Female.vs.SJ28Male & sja-miR-36-5p & 6.064890382 & 2.868103622 & 1.080384422 & 0.1626144 & \\
\hline SJ28Female.vs.SJ28Male & sja-miR-61 & 197.1678198 & 351.9163145 & -0.83580829 & 0.1942248 & \\
\hline SJ28Female.vs.SJ28Male & sja-miR-71a & 2544.398163 & 2175.743408 & 0.225816025 & 0.6163959 & \\
\hline SJ28Female.vs.SJ28Male & sja-miR-71b-3p & 2.679150606 & 3.513426937 & -0.39110321 & 0.6704208 & \\
\hline SJ28Female.vs.SJ28Male & sja-miR-71b-5p & 586.0568347 & 178.0375324 & 1.718859171 & 0.1803544 & \\
\hline SJ28Female.vs.SJ28Male & sja-miR-7-3p & 0.235529724 & 0.932133677 & -1.98462773 & 0.3297433 & \\
\hline SJ28Female.vs.SJ28Male & sja-miR-7-5p & 0.235529724 & 0.358512953 & -0.60611611 & 0.8476834 & \\
\hline SJ28Female.vs.SJ28Male & sja-miR-8-3p & 33.18024981 & 96.87019985 & -1.54572817 & 0.166735 & \\
\hline
\end{tabular}


(the highest co-expression of female and male in 28 days), sja-miR-36-3p (the highest differential expression of female and male in 14 days), as well as sja-miR-3505 (the highest differential expression of female and male in 28 days). The sequences, as well as the melt curves of the four miRNAs, are presented in Table 9 and Figure 3. The novel miRNAs identified in the present study may clarify the development of $S$. japonicum, thus furthering the understanding of the biology of this trematode.

Table 9. Sequences in Real-time quantitative PCR verification of co-expression and differential miRNA in male and female adult worm of Schistosomulum and Schistosoma japonicum

\begin{tabular}{lll}
\hline miRNA & Sequence & Expression stage \\
\hline sja-miR-125b & TCCCTGAGACTGATAATTGC & $\begin{array}{l}\text { the highest co-expression of female and male } \\
\text { in } 14 \text { days } \\
\text { the highest co-expression of female and male } \\
\text { in 28 days }\end{array}$ \\
sja-miR-36-3p & CACCGGGTAGACATTCATTCGC & $\begin{array}{l}\text { the highest differential expression of female } \\
\text { and male in } 14 \text { days }\end{array}$ \\
sja-miR-3505 & TGACTGTCTGGACTCAGTAGCT & $\begin{array}{l}\text { the highest differential expression of female } \\
\text { and male in } 28 \text { days }\end{array}$ \\
\hline
\end{tabular}

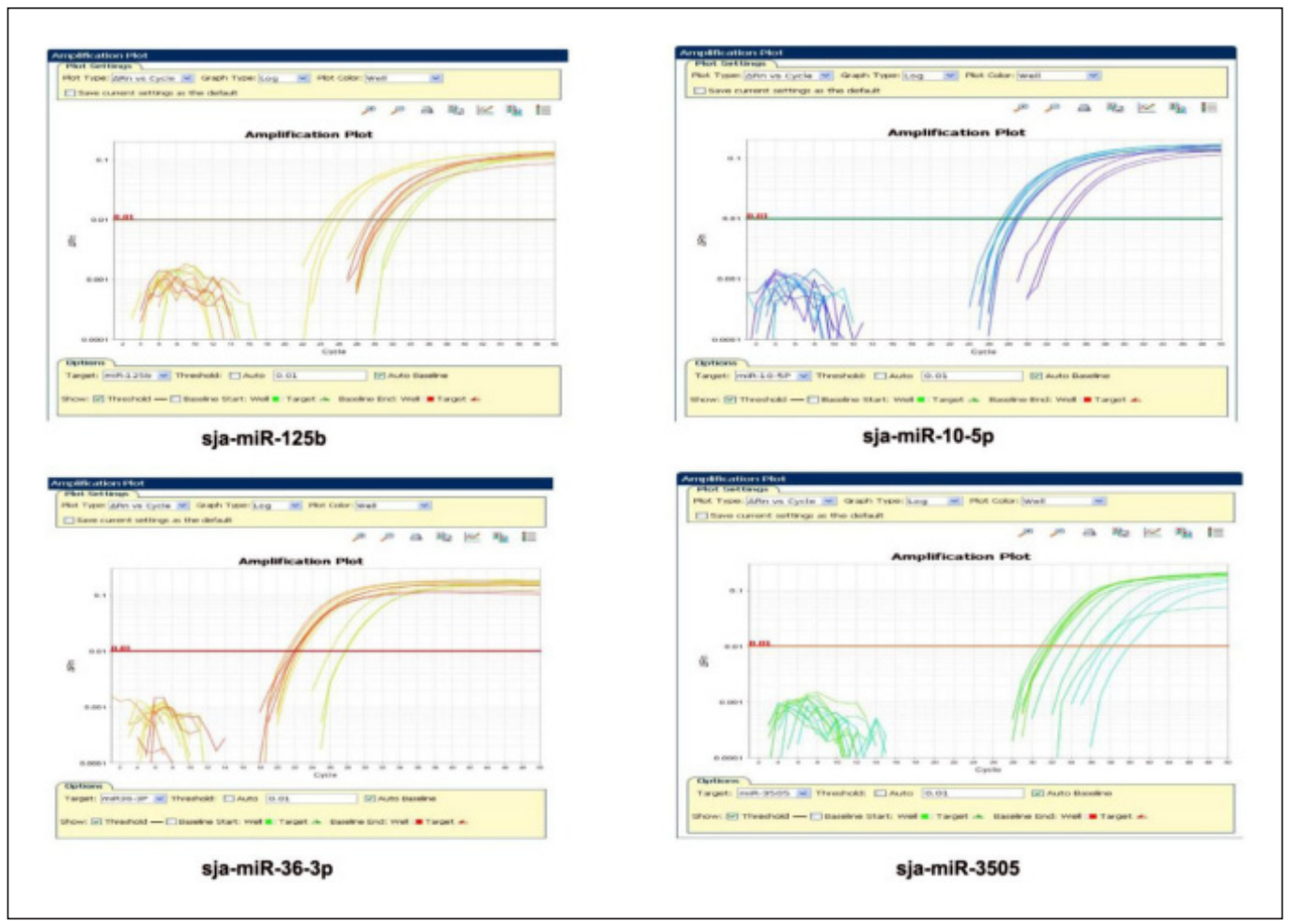

Figure 3. Real-time quantitative PCR verification of co-expression and differential miRNA in male and female adult worm of Schistosomulum and Schistosoma japonicum. Melt curves of four miRNAs and endogenous control (18S) is shown. 


\section{DISCUSSION}

MicroRNAs are a class of small non-coding RNAs that have an important role in the transcriptional and post-transcriptional regulation of genes in both plants and animals (Liu et al., 2017; Masuda et al., 2017; Zhao et $a l ., 2018)$. Over the last decade, an increasing number of miRNAs have been identified in S. japonicum, some of which regulate cellular differentiation, sexual maturation and metabolism of the trematode (Cai $\mathrm{et} \mathrm{al.,}$ 2011, 2013; Sun et al., 2014; Zhu et al., 2016)

During the development of $S$. japonicum, the sexual differentiation starts approximately 14 dpi infection, while egg-laying starts approximately 28 days post-infection (He \& Yang, 1980). In terms of the published criteria for distinguishing bilaterian miRNAs from other types of small RNAs (Ruby et al., 2006, 2007; Grimson et al., 2008), it can provide insight into the mechanism underlying $S$. japonicum development, differentiation and maturation by comparing the miRNA profiles in female and male worms at 14 dpi and 28 dpi.

In this study, we examined the expression profile of miRNAs in individual $S$. japonicum of different sex before and after pairing (18 and $24 \mathrm{dpi}$ ). The majority of differential expressed miRNAs were highly abundant at 14 dpi, except for sja-miR-125b and sja-miR3505 , in both male and female (Mark: begin to be paired). Moreover, it was estimated that sja-miR-125b and sja-miR-3505 might be related to laying eggs. Besides, we found three female-biased expressions and 15 male-biased expressions in miRNAs at $14 \mathrm{dpi}$ and $28 \mathrm{dpi}$; the function of 3 female-biased expressed miRNA was related to eggs laying, while 15 male-biased expressions were associated with sexual maturity.

In previous studies, let-7 has shown to be essential for the proliferation, differentiation, and apoptosis in cells (Crippa et al., 2012; Shimono et al., 2015; Jin et al., 2016). Studies suggested that sja-let-7 take part in the transformation from miracidium to sporocyst in the snail intermediate host (Huang et al., 2009). Moreover, sja-let-7 participates in chromosome transmission fidelity protein 18 , large subunit ribosomal protein L21e and
N-acetyltransferase 10 (Sun et al., 2014). In this study, we found that sja-let-7 was highlyexpressed at 14 dpi in both males and females, which suggested that this miRNA has an important role in sexual maturation of S. japonicum.

Bantam are known as regulators for both proliferation and apoptosis that target the proapoptotic gene hid (Banerjee et al., 2017; Wu et al., 2017; Brennecke et al., 2003). Moreover, predicted target genes of sjabantam participate in ATP-dependent RNA helicase DDX17, ATP-dependent DNA helicase PIF1 and chromodomain-helicaseDNA-binding protein 7 (Sun et al., 2015). In this study, sja-bantam was expressed at different stages in males and females. At the same time, the highest expression levels were observed at $28 \mathrm{dpi}$ in females as the report description on the function in ovary development (Zhu et al., 2016).

Using a KEGG pathway analysis, we found a significant difference in the expression of miRNAs. We found that the predicted target genes of sja-miR-2a-5p and sja-miR-3484-5p, which were expressed at 14 dpi in males, were significantly clustered in DNA topoisomerase III, Rap guanine nucleotide exchange factor 1 and L-serine/Lthreonine ammonia-lyase. Target genes of sja-miR-2d-5p, sja-miR-31-5p and sja-miR125a, which were expressed at $14 \mathrm{dpi}$ in males and particularly females, were clustered in kelch-like protein 12, fructosebisphosphate aldolase, class I, and heat shock protein $90 \mathrm{kDa}$ beta. Liu et al. (2009) have reported that HSP90 was related to reproductive development. Predicted target genes of sja-miR-3483-3p (expressed at 28 dpi in female without in the 28 dpi male), were significantly clustered in 26S proteasome regulatory subunit N1, ATP-dependent RNA helicase DDX17. Predicted target genes of sja-miR-219-5p, which were differentially expressed at $28 \mathrm{dpi}$ in females and particularly males, were clustered in DNA excision repair protein ERCC-6, protein phosphatase 1D, and ATPase family AAA domain-containing protein 3A/B. At 28 dpi, eight miRNAs were significantly up-regulated in females compared to males, and the predicted target genes of these miRNAs were 
significantly clustered in heat shock protein $90 \mathrm{kDa}$ beta, 26S proteasome regulatory subunit $\mathrm{N} 1$, and protein arginine $\mathrm{N}$ methyltransferase 1 .

\section{CONCLUSIONS}

The miRNAs research on $S$. japonicum may have an essential role in clarifying this trematode's growth, development, maturation and infection ability to mammalian hosts in its complex life cycle. These data may be helpful for developing new drug targets and vaccine candidates for schistosomiasis.

\section{Conflicts of interest}

The authors declare no conflicts of interest with regards to this study or the manuscript prepared for publication.

Acknowledgments. This study was supported by the General Program Shanghai Municipal Commission of Health and Family Planning of China (Grant No. 201840286 and 201640278), the Program for the Shanghai Natural Science Foundation of China (Grant No. 18ZR1443500), the Youth Science Foundation of Chinese Center for Disease Control and Prevention (Grant No. 2018A105), the Shenzhen San-Ming Project for prevention and research on vectorborne diseases (SZSM201611064), the National Sharing Service Platform for Parasite Resources (TDRC-22), the Program for National Key Research and Development Program of China (Grant No. 2016YFC1202000, 2016YFC1202005, and 2016YFC1202700), the Program for the National Science and Technology Major Program (Grant No. 2012ZX10004-220 and 2018ZX10734-404), the Chinese Special Program for Scientific Research of Public Health (Grant No. 201502021 and 201202019), the National Key Technology R\&D Program (Grant No. 2008BAI56B03), the Fourth Round of Three-Year Public Health Action Plan of Shanghai, China (Grant No. 15GWZK0101), the Open project of Key Laboratory of Tropical Disease Control (Sun Yat-sen University), Ministry of Education (Grant No. 2019kfkt02), and the Parasitic and Tropical
Diseases Resource Center Project of National Science and Technology Basic Conditions Platform Program, Key Laboratory of Echinococcosis Prevention and Control (Project No. 2020WZK2002)

\section{REFERENCES}

Ai, L., Xu, M.J., Chen, M.X., Zhang, Y.N., Chen, S.H., Guo, J., Cai, Y.C., Zhou, X.N., Zhu, X.Q. \& Chen, J.X. (2012). Characterization of microRNAs in Taenia saginata of zoonotic significance by Solexa deep sequencing and bioinformatics analysis. Parasitology Research 110: 2373-2378.

Altschul, S.F., Gish, W., Miller, W., Myers, E.W., \& Lipman, D.J. (1990). Basic local alignment search tool. Journal of Molecular Biology 215: 403-410.

Banerjee, A. \& Roy, J.K. (2017). Study of bantam miRNA expression in brain tumour resulted due to loss of polarity modules in Drosophila melanogaster. Journal of Genetics 96: 365-369.

Brennecke, J., Hipfner, D.R., Stark, A., Russell, R.B. \& Cohen, S.M. (2003). Bantam encodes a developmentally regulated microRNA that controls cell proliferation and regulates the proapoptotic gene hid in Drosophila. Cell 113: 25-36.

Bergman, C.M. \& Quesneville, H. (2007). Discovering and detecting transposable elements in genome sequences. Briefings in Bioinformatics 8: 382-392.

Cai, P., Hou, N., Piao, X., Liu, S., Liu, H., Yang, F., Wang, J., Jin, Q., Wang, H. \& Chen, Q. (2011). Profiles of small non-coding RNAs in Schistosoma japonicum during development. PLoS Neglected Tropical Diseases 5: e1256.

Cai, P., Piao, X., Hao, L., Liu, S., Hou, N., Wang, H. \& Chen, Q. (2013). A deep analysis of the small non-coding RNA population in Schistosoma japonicum eggs. PLoS One 8: e64003.

Chen, J., Yang, Y., Guo, S., Peng, J., Liu, Z., Li, J., Lin, J. \& Cheng, G. (2010). Molecular cloning and expression profiles of argonaute proteins in Schistosoma japonicum. Parasitology Research 107: 889-899. 
Cheng, G. \& Jin, Y. (2012). MicroRNAs: potentially important regulators for schistosome development and therapeutic targets against schistosomiasis. Parasitology 139: 669-679.

Crippa, S., Cassano, M. \& Sampaolesi, M. (2012). Role of miRNAs in muscle stem cell biology: proliferation, differentiation and death. Current Pharmaceutical Design 18: 1718-1729.

Gardner, P.P., Daub, J., Tate, J., Moore, B.L., Osuch, I.H., Griffiths-Jones, S., Finn, R.D., Nawrocki, E.P., Kolbe, D.L., Eddy, S.R. \& Bateman, A. (2011). Rfam: Wikipedia, clans and the "decimal" release. Nucleic Acids Research 39 (Database issue): D141-D145.

Gray, D.J., McManus, D.P., Li, Y., Williams, G.M., Bergquist, R. \& Ross, A.G. (2010). Schistosomiasis elimination: lessons from the past guide the future. The Lancet Infectious Diseases 10: 733-736.

Grimson, A., Srivastava, M., Fahey, B., Woodcroft, B.J., Chiang, H.R., King, N., Degnan, B.M., Rokhsar, D.S. \& Bartel, D.P. (2008). Early origins and evolution of microRNAs and Piwi-interacting RNAs in animals. Nature 455: 1193-1197.

Hao, L., Cai, P., Jiang, N., Wang, H. \& Chen, Q. (2010). Identification and characterization of microRNAs and endogenous siRNAs in Schistosoma japonicum. BMC Genomics 11: 55 .

He, Y.X. \& Yang, H.Z. (1980). Physiological studies on the post-cercarial development of Schistosoma japonicum. ACTA Zoologica Sinica 26: 32-41.

Huang, J., Hao, P., Chen, H., Hu, W., Yan, Q., Liu, F. \& Han, Z.G. (2009). Genome-wide identification of Schistosoma japonicum microRNAs using a deep-sequencing approach. PLoS One 4: e8206.

Jin, B., Wang, W., Meng, X.X., Du, G., Li, J., Zhang, S.Z., Zhou, B.H. \& Fu, Z.H. (2016). Let-7 inhibits self-renewal of hepatocellular cancer stem-like cells through regulating the epithelial-mesenchymal transition and the Wnt signaling pathway. BMC Cancer 16: 863.

Kozomara, A. \& Griffiths-Jones, S. (2011). miRBase: integrating microRNA annotation and deep-sequencing data. Nucleic
Acids Research 39 (Database issue): D152-D157.

Lewis, B.P., Shih, I.H., Jones-Rhoades, M.W., Bartel, D.P. \& Burge, C.B. (2003). Prediction of mammalian microRNA targets. Cell 115: 787-798.

Li, S.Z., Zheng, H., Abe, E.M., Yang, K., Bergquist, R., Qian, Y.J., Zhang, L.J., Xu, Z.M., Xu, J., Guo, J.G., Xiao, N. \& Zhou, X.N. (2014). Reduction patterns of acute schistosomiasis in the People's Republic of China. PLoS Neglected Tropical Diseases 8: e2849.

Liu, F., Cui, S.J., Hu, W., Feng, Z., Wang, Z.Q. \& Han, Z.G. (2009). Excretory/secretory proteome of the adult developmental stage of human blood fluke, Schistosoma japonicum. Molecular \& Cellular Proteomics: MCP 8: 1236-1251.

Liu, W., Ma, R. \& Yuan, Y. (2017), Posttranscriptional Regulation of Genes Related to Biological Behaviors of Gastric Cancer by Long Noncoding RNAs and MicroRNAs. Journal of Cancer 8: 4141-4154.

Masuda, T., Hayashi, N., Kuroda, Y., Ito, S., Eguchi, H. \& Mimori, K. (2017). MicroRNAs as Biomarkers in Colorectal Cancer. Cancers (Basel) 9. pii: E124.

Mount, D.W. (2007). Using the Basic Local Alignment Search Tool (BLAST). $\mathrm{CSH}$ Protoc 2007: pdb.top17.

Mourão, M.M., Grunau, C., LoVerde, P.T., Jones, M.K. \& Oliveira, G. (2012). Recent advances in Schistosoma genomics. Parasite Immunology 34: 151-162.

PLOS Pathogens Staff (2016). Correction: MicroRNAs Are Involved in the Regulation of Ovary Development in the Pathogenic Blood Fluke Schistosoma japonicum. PLoS Pathogens 12: e1005582.

Ruby, J.G., Jan, C., Player, C., Axtell, M.J., Lee, W., Nusbaum, C., Ge, H. \& Bartel, D.P. (2006). Large-scale sequencing reveals 21U-RNAs and additional microRNAs and endogenous siRNAs in C. elegans. Cell 127: 1193-1207.

Ruby, J.G., Stark, A., Johnston, W.K., Kellis, M., Bartel, D.P. \& Lai, E.C. (2007). Evolution, biogenesis, expression, and target predictions of a substantially 
expanded set of Drosophila microRNAs. Genome Research 17: 1850-1864.

Siqueira, L.D.P., Fontes, D.A.F., Aguilera, C.S.B., Timóteo, T.R.R., Ângelos, M.A., Silva, L.C.P.B.B., de Melo, C.G., Rolim, L.A., da Silva, R.M.F. \& Neto, P.J.R. (2017). Schistosomiasis: Drugs used and treatment strategies. Acta Tropica 176: 179-187.

Shimono, Y., Mukohyama, J., Nakamura, S. \& Minami, H. (2015). MicroRNA Regulation of Human Breast Cancer Stem Cells. Journal of Clinical Medicine 5. pii: E2.

Song, L.G., Wu, X.Y., Sacko, M. \& Wu, Z.D. (2016). History of schistosomiasis epidemiology, current status, and challenges in China: on the road to schistosomiasis elimination. Parasitology Research 115: 4071-4081.

Sun, J., Hu, W. \& Li, C. (2013). Beyond heme detoxification: a role for hemozoin in iron transport in S. japonicum. Parasitology Research 112: 2983-2990.

Sun, J., Wang, S., Li, C., Ren, Y. \& Wang, J. (2014). Novel expression profiles of microRNAs suggest that specific miRNAs regulate gene expression for the sexual maturation of female Schistosoma japonicum after pairing. Parasites \& Vectors 7: 177.

Sun, J., Wang, S.W. \& Li, C. (2015). ATP synthase: an identified target gene of bantam in paired female Schistosoma japonicum. Parasitology Research 114: 593-600.

Sun, L.P., Wang, W., Hong, Q.B., Li, S.Z., Liang, Y.S., Yang, H.T. \& Zhou, X.N. (2017). Approaches being used in the national schistosomiasis elimination programme in China: a review. Infectious Diseases of Poverty 6: 55.

Tebeje, B.M., Harvie, M., You, H., Loukas, A. \& McManus, D.P. (2016). Schistosomiasis vaccines: where do we stand? Parasites \& Vectors 9: 528.

The Carter Center (2014). Schistosomiasis control program. http://www.carter center.org/health/schistosomiasis/ index.html
Walker, A.J. (2011). Insights into the functional biology of schistosomes. Parasites \& Vectors 4: 203.

Wang, W., Wang, L. \& Liang, Y.S. (2012). Susceptibility or resistance of praziquantel in human schistosomias is: are view. Parasitology Research 111: 1871-1877.

WHO (2016) Schistosomiasis. http://www. Who.Int/mediacentre/factsheets/fs115/ en/

Wei, Y., Chen, S., Yang, P., Ma, Z. \& Kang, L. (2009). Characterization and comparative profiling of the small RNA transcriptomes in two phases of locust. Genome Biology 10: R6.

Wu, Y.C., Lee, K.S., Song, Y., Gehrke, S. \& Lu, B. (2017). The bantam microRNA acts through Numb to exert cell growth control and feedback regulation of Notch in tumor-forming stem cells in the Drosophila brain. PLoS Genetics 13 : e1006785.

Xu, M.J., Ai, L., Fu, J.H., Nisbet, A.J., Liu, Q.Y., Chen, M.X., Zhou, D.H. \& Zhu, X.Q. (2012). Comparative characterization of microRNAs from the liver flukes Fasciola gigantica and F. hepatica. PLoS One 7: e53387.

Xue, X., Sun, J., Zhang, Q., Wang, Z., Huang, Y. \& Pan, W. (2008). Identification and characterization of novel microRNAs from Schistosoma japonicum. PLoS One 3: e4034.

Zhao, Y., Cong, L. \& Lukiw, W.J. (2018). Plant and Animal microRNAs (miRNAs) and Their Potential for Inter-kingdom Communication. Cell and Molecular Neurobiology 38: 133-140.

Zhu, L., Zhao, J., Wang, J., Hu, C., Peng, J., Luo, R., Zhou, C., Liu, J., Lin, J., Jin, Y., Davis, R.E. \& Cheng, G. (2016). MicroRNAs are involved in the regulation of ovary development in the pathogenic blood fluke Schistosoma japonicum. PLoS Pathogens 12: e1005423.

Zuker, M. (2003). Mfold web server for nucleic acid folding and hybridization prediction. Nucleic Acids Research 31: 3406-3415. 